\title{
Una aproximación al término de calidad en la educación superior
}

\author{
An approach to the term quality in superior education
}

\begin{abstract}
Resumen
Una de las tareas más difíciles es definir la calidad. El término ha ido en evolución a lo largo del último siglo hasta el presente. No hay un consenso para su conceptualización, sin embargo ha sido una búsqueda constante para las organizaciones de diversa índole y, más recientemente, en el ámbito educativo. El énfasis en la calidad en educación superior demanda una revisión de su definición entre los diferentes actores del ámbito universitario para poder mejorar los procesos educativos. La medición de la calidad es otro aspecto que merece atención por la diversidad de enfoques y por lo dificultoso que resulta definir los parámetros de evaluación. La evaluación de la calidad educativa puede ser interna cuando la institución evalúa sus procesos académicos o los relacionados a estos, y por otro lado, la evaluación externa que permite acceder a la acreditación de la carrera o institución como un reconocimiento de la calidad. Diversos modelos de calidad internacionales coinciden en los aspectos que deben ser evaluados en las instituciones: Proyecto educativo, estudiantes, profesores, procesos académicos, investigación, visibilidad nacional e internacional, egresados, bienestar institucional, organización, administración y gestión, recursos físicos y financieros.

El modelo peruano de acreditación está basado en un enfoque de procesos inicialmente aplicado al ámbito empresarial y es labor de la universidad peruana adaptarse a los cambios demandados para elevar la calidad educativa.
\end{abstract}

Palabras clave: Educación superior, acreditación, calidad de la educación.

\begin{abstract}
One of the most difficult tasks is to define quality. The term has been evolution over the last century to the present. There is no consensus for its conceptualization; however it has been a constant search for diverse organizations and more recently, in the field of education.

The emphasis on quality in superior education demands a revision of its definition among different stakeholders from academia to improve educational processes. The measurement of quality is another aspect that deserves attention by the diversity of approaches and therefore difficult to define the parameters for evaluation. The evaluation of educational quality can be internal when the institution evaluates your processes academic or related to these, and on the other hand, the external evaluation that allows access to the accreditation of the degree program or institution as recognition of the quality.

Various international quality models coincide in the aspects that should be evaluated in the institutions: Educational Project, Students, Teachers, Academic Processes, Research, National and International Visibility, Graduated, Institutional well-being, Organization, Administration and Management, Physical and Financial Resources

The Peruvian model accreditation is based on a process approach initially applied to the business and work of the Peruvian university adapt to changes in demand for raising educational quality.

Keywords: Superior education; accreditation; education quality
\end{abstract}

\section{Introducción}

El Sistema Nacional de Evaluación, Acreditación y Certificación de la Calidad Educativa (Sineace) es el conjunto de organismos, normas y procedimientos estructurados e integrados funcionalmente, destinados a definir y establecer los criterios, estándares y procesos de evaluación, acreditación y certificación a fin de asegurar los niveles básicos de calidad que deben brindar las instituciones a las que se refiere la Ley General de Educación N ${ }^{\circ}$ 28044, y promover su desarrollo cualitativo. Fue creado por ley el 19 de mayo del $2006^{1}$.
En nuestro país, el proceso de acreditación de la calidad en las instituciones de educación superior es voluntario ${ }^{2}$; sin embargo, las carreras que pretenden demostrar la calidad de sus programas se someten a este proceso.

Un punto de partida es establecer qué se entiende por calidad educativa. El artículo pretende realizar una breve revisión del término y cómo es que se pretende evaluar la calidad en educación superior. Para tal propósito, se ha realizado una revisión de artículos publicados a nivel nacional e internacio-

\section{Artículo de Revisión} 1. Facultad de Estomatología de la Universidad
Peruana Cayetano Heredia, Perú.

Correspondencia

C.D. Milagros Carrasco Loyola

Especialista en Salud Pública Estomatológica.

Jr. Samiria 283 Torre 6 Departamento 802.

Lima 34, Perú.

Correo electrónico:

milagrosbcarrasco@yahoo.com
Recibido: 25-03-15

Aceptado: 30-10-15 nal sobre el tema, que nos aproximen a conceptualizar la calidad educativa y su evaluación.

\section{Calidad y calidad en educación}

El término calidad está en constante evolución, en continua adaptación a las necesidades de los grupos sociales. La mayoría de autores distinguen seis etapas en el desarrollo y evolución de la calidad ${ }^{3}$. Estas etapas son esquematizadas en la tabla 1.

Existen múltiples definiciones para el término calidad. En los últimos años concita la atención e interés de la in- 
dustria, administración y educación. En educación superior ha sido difícil poder definirla o llegar a un consenso, pese a la presión que reciben para elevar la calidad de la enseñanza.

A comienzos de los noventa, el tema de la calidad se traslada al campo de la educación, específicamente en sus inicios a la educación superior y se vuelve un desafío o problemática en la Comunidad Europea, norteamérica y latinoamérica. Todos envueltos en orientar la calidad a la eficiencia y eficacia de los procesos educativos en la formación profesional ${ }^{4}$.

Etimológicamente, calidad y cualidad tienen la misma raíz latina (qualitas, qualitatis) y así puede decirse que son las cualidades las que determinan la calidad de algo o alguien. En este sentido, se afirma que la calidad no es una propiedad discreta sino la suma y concierto de propiedades cuyo valor es convencional, así como su número 5 . Una enseñanza de calidad es la que logra un aprendizaje profundo por parte de los estudiantes y alcanza las metas establecidas para este nivel ${ }^{6}$, asociadas a las competencias e incluso con la infraestructura, tecnología y servicios ${ }^{7}$.

La Ley General de Educación $N^{\circ}$ 28044, en su artículo 13, especifica que la calidad de la educación es el nivel óptimo de formación que deben alcanzar las personas para enfrentar los retos del desarrollo humano, ejercer su ciudadanía y continuar aprendiendo durante toda la vida ${ }^{8}$. La calidad, dentro del marco legal, se define como el conjunto de características inherentes a un producto o servicio que cumple con los requisitos para satisfacer las necesidades preestablecidas ${ }^{9}$.

Kent, citado por Salas ${ }^{10}$, define cuatro conceptos diferentes de la calidad:

- Como lo excepcional, que ha sido el concepto académico tradicional.

- En la consistencia de un producto, que es la conceptualización propia de la producción industrial.

- En el cumplimiento de una misión, que es el concepto gerencial, que se enmarca en el análisis de la eficiencia y eficacia del cumplimiento de una tarea o misión.

- En la satisfacción de las necesidades del usuario, que es el que se emplea en el mercado.

- En la transformación del hombre, que es el concepto educacional o pedagógico contemporáneo de formación de los individuos y grupos sociales.
Para Yzaguirre ${ }^{11}$, hablar de calidad de la educación incluye varias dimensiones o enfoques, complementarios entre sí. La primera dimensión es la eficacia: una educación de calidad es aquella que logra que los alumnos realmente aprendan lo que se supone deben aprender, aquello que está establecido en los planes y programas curriculares, al cabo de determinados ciclos o niveles. En esta perspectiva, el énfasis está puesto en que, además de asistir a clase, los estudiantes aprendan en su paso por el sistema. Esta dimensión del concepto pone en primer plano los resultados de aprendizaje efectivamente alcanzados por la acción educativa. Una segunda dimensión del concepto de calidad, complementaria de la anterior, está referida a qué es lo que se aprende en el sistema y a su pertinencia en términos individuales y sociales. En este sentido, una educación de calidad es aquella cuyos contenidos responden adecuadamente a lo que el individuo necesita para desarrollarse como persona -intelectual, afectiva, moral y físicamente-, y para actuar en los diversos ámbitos de la sociedad -el político, el económico, el social-. Esta dimensión del concepto pone en primer plano los fines atribuidos a la acción educativa y su realización en los diseños y contenidos curriculares. Finalmente, una tercera dimensión es la que se refiere a los procesos y medios que el sistema brinda a los alumnos para el desarrollo de su experiencia educativa. Desde esta perspectiva, una educación de calidad es aquella que ofrece al estudiante un adecuado contexto físico para el aprendizaje, un cuerpo docente convenientemente preparado para la tarea de enseñar, buenos materiales de estudio y de trabajo, estrategias didácticas adecuadas, etc. Esta dimensión del concepto pone en primer plano el análisis de los medios empleados en la acción educativa.

Puede definirse la calidad entonces desde diferentes enfoques. Si la definimos en función del producto, la calidad puede ser juzgada por el desempeño de sus estudiantes o en las pruebas finales de la carrera. Si el enfoque es en el usuario, es decir el estudiante, la calidad sería entendida como aquella que satisface las expectativas del estudiante, se orienta hacia el logro de las competencias requeridas para su inserción en el mercado laboral, principalmente. Así mismo, si la calidad está orientada al valor, a la eficiencia, se entiende la calidad como aquella que logra sus objetivos con uso eficiente de sus recursos e infraestructura.
No hay un acuerdo universal sobre lo que es calidad, pero cada vez se mide más con dos aspectos ${ }^{12}$ : (a) formación de las personas que terminan el programa, y (b) capacidad de la institución para producir cambios que mejoren esa formación, y la planificación de ese cambio, así como la operativización de estrategias para el cambio institucional. La educación no es meramente adquisición de conocimientos, sino también de herramientas, educación multicultural, uso de tecnologías, pensamiento crítico y capacidad de aprender temas nuevos, después de haber obtenido el título.

Vidal ${ }^{7}$ propone que la calidad educativa ha de ser caracterizada, entre otros aspectos, por:

\section{- Ser accesible.}

- Facilitar los recursos personales, organizativos y materiales requeridos.

- Promover cambio e innovación a través de la reflexión compartida sobre la propia práctica docente y el trabajo colaborativo del profesorado.

- Promover la participación activa del alumnado, tanto en el aprendizaje como en la vida de la institución, en un marco de valores donde todos se sientan respetados y valorados como personas.

- Lograr la participación de las familias e insertarse en la comunidad.

- Estimular y facilitar el desarrollo y el bienestar del profesorado, así como de los demás profesionales de la institución.

La Organización de las Naciones Unidas para la Educación, la Ciencia y la Cultura (Unesco) refiere en el artículo 11 de la Declaración Mundial sobre la Educación Superior en el siglo XXI que la calidad de la enseñanza superior es un concepto pluridimensional que debería comprender todas sus funciones y actividades: enseńanza y programas académicos, investigación y becas, personal, estudiantes, edificios, instalaciones, equipamiento y servicios a la comunidad y al mundo universitario. Considera como una característica más a la dimensión internacional: el intercambio de conocimientos, la creación de sistemas interactivos, la movilidad de profesores y estudiantes y los proyectos de investigación internacionales, aun cuando se tengan debidamente en cuenta los valores culturales y las situaciones nacionales. Dicho en otras pala- 
bras, si bien es cierto que se toman en cuenta los requerimientos o competencias del medio en el cual se desarrolla el estudiante, también debe considerarse la demanda del mercado internacional, de modo que el egresado pueda responder a otras regiones del país y del mundo ${ }^{13}$.

La calidad en Educación para Cuya ${ }^{14}$ es la suma de cuatro aspectos no excluyentes: (a) grado del logro del perfil, competencias y los objetivos educacionales del nivel en que se estudia, (b) infraestructura, (c) tecnología y (d) servicios. Una característica importante de la calidad en educación superior es el factor transformador del propio proceso educativo en todos los involucrados, no solo en el estudiante, de forma tal que ejerce un efecto benéfico multiplicador hacia la sociedad, no siempre perceptible o medible. Implica valores, atención de problemas de individuos y la sociedad, contribución al desarrollo científico y tecnológico del país y el bienestar común. Para Vidal, son los efectos positivamente valorados por la sociedad respecto del proceso de formación que llevan a cabo las personas en su cultura ${ }^{7}$.

Lo que es calidad para una institución no necesariamente lo es para otra. Es difícil generalizarla a todo un país o región. La aproximación al concepto de calidad varía según el momento, el lugar, las personas, entre otros, y esto implica una relatividad en su definición misma.

Para el sistema de aseguramiento de la calidad, las normas de la International Organization for Standardization (Organización Internacional de Normalización), ISO, establecen los requisitos que deben cumplir las empresas para satisfacer a los clientes ${ }^{15}$. Su principal objetivo es garantizar la calidad del producto y/o servicio prestado, según una metodología definida de trabajo y a unos ensayos registrados que prueben el correcto desarrollo de las tareas y sirvan para analizar toda la información registrada y, de acuerdo a ella, actuar para corregir los errores ${ }^{16}$.

Son múltiples las ventajas: la reducción de los costos, el aumento de la eficacia y el tiempo de respuesta, la reducción de quejas y reclamos, la motivación de los empleados y la mayor claridad en la distribución de las responsabilidades, así como el prestigio al superar las auditorías de las empresas certificadoras. Sin embargo, para otros implica papeleo y burocracia, formalismo y costo ${ }^{16}$.
Su adaptación al ámbito de la educación superior implica definir a la Universidad y sus funciones de docencia, investigación, extensión y proyección social. Es ver a la institución como empresa, al estudiante como cliente, al proceso educativo como servicio, a las funciones sustanciales como sistemas y procesos. No hay la seguridad que el sistema de aseguramiento de la calidad sea la solución a todos los problemas de la institución, pero si supone un camino de mejora que facilita ser más competitivos y por ello tener más posibilidades $y$ perspectivas de futuro ${ }^{16}$.

\section{Medición de la calidad}

Un punto interesante es cómo medir la calidad. Para determinar si algo tiene calidad es imprescindible obtener un consenso, más o menos amplio, sobre cuántas y cuáles son las cualidades que habrán de evaluarse ${ }^{5}$.

Según la Red Iberoamericana de Acreditación de la Calidad de la Educación Superior, para medirse adecuadamente suele implicar la evaluación de la docencia, el aprendizaje, la gestión, y los resultados obtenidos. Cada parte puede ser medida por su calidad y el conjunto supone la calidad global ${ }^{17}$.

La evaluación de la calidad ha pasado desde la mirada desde dentro de la institución hacia una evaluación externa que reduce la subjetividad de los análisis ${ }^{18}$. Una mirada desde fuera lo constituyen los rankings universitarios, los cuales tienen por objeto realizar una jerarquización de las instituciones de educación superior basándose en sistemas de indicadores que intentan valorar el nivel de calidad de las diferentes instituciones, en un esfuerzo por facilitar dicha información a la sociedad. Actualmente existe un gran número de rankings a nivel nacional e internacional de las universidades. Cada uno de ellos se elabora con una metodología particular, por lo que no son comparables entre ellos ${ }^{19}$.

El hecho de que cada vez exista un mayor número de evaluaciones mundiales de educación superior indica que su influencia aumenta y que están siendo progresivamente más utilizados ${ }^{19}$. Por otro lado, este tipo de comparaciones tiende a poner énfasis sobre la investigación y a usar los resultados de esta como medida estándar del valor de cada institución ${ }^{20}$.

Pires y col. ${ }^{21}$ hacen mención a una distinción importante que suele hacerse entre procesos internos, los cuales son generados y aplicados por las propias instituciones, y los procesos externos, más comúnmente asociados al licenciamiento, evaluación o acreditación. En el caso de los procesos externos, estos suelen agruparse de acuerdo a los propósitos buscados. Si el propósito principal es controlar la calidad, es decir, asegurar que solo operen instituciones que satisfagan criterios mínimos de calidad, elinstrumento utilizado es el licenciamiento, es decir, la autorización y reconocimiento de instituciones, carreras o programas. Si lo que se busca es garantizar la calidad de una institución o programa, el instrumento más apropiado es la acreditación, mediante la cual se verifica el cumplimiento de ciertos estándares o criterios públicamente conocidos.

Por otro lado, si la finalidad principal es el desarrollo de procesos de mejora continua de las instituciones, sus carreras y programas, el principal instrumento es la auditoría académica, centrada en la evaluación de los procesos internos de aseguramiento de la calidad. En todos estos casos, se utiliza la evaluación interna o autoevaluación, como elemento de base, y la evaluación externa como un mecanismo de verificación ${ }^{21}$.

Los aspectos que deben considerarse cuando se evalúa calidad, propuestos por el Consejo Nacional de Acreditación de Colombia, ${ }^{22}$ son:

a) La incorporación de profesores con altos niveles de cualificación y con modalidades de vinculación apropiadas, que lideren los procesos académicos.

b) La investigación científica, tecnológica, humanística y artística en sintonía con el saber universal y con alta visibilidad.

c) La formación integral de las personas hacia el desarrollo de la capacidad de abordar con responsabilidad ética, social y ambiental los retos de desarrollo endógeno y participar en la construcción de una sociedad más incluyente.

d) La pertinencia y relevancia social que supone ambientes educativos más heterogéneos y flexibles, en perspectiva de responder adecuadamente a los requerimientos formativos y de investigación de los respectivos entornos.

e) El seguimiento a egresados que permita validar el proceso formativo y un adecuado aporte al programa de sus experiencias profesionales. 
f) La generación de sistemas de gestión transparentes, eficaces y eficientes que garanticen los derechos y los deberes de las personas.

g) La internacionalización, con todo lo que ello implica, como movilidad de profesores y estudiantes, reconocimientos académicos trasnacionales, redes, alianzas multinacionales, publicaciones conjuntas, entre otras.

h) Los procesos formativos flexibles e interdisciplinarios sustentados en un trabajo de créditos académicos y el desarrollo de competencias, especialmente actitudes, conocimientos, capacidades y habilidades.

i) Los recursos físicos y financieros adecuados y suficientes.

Diversos modelos de acreditación ${ }^{22-25}$ refieren los siguientes aspectos para evaluar la calidad educativa: proyecto educativo, estudiantes, profesores, procesos académicos, investigación, visibilidad nacional e internacional, egresados, bienestar institucional, organización, administración y gestión, recursos físicos y financieros.

La Unesco refiere que la calidad no puede ser medida solo por parámetros cuantitativos, tales como ratios estudiante/profesor, capacidad de ambientes de lectura, laboratorios, bibliotecas, entre otros. Los parámetros cualitativos como competencias académicas y pedagógicas de los docentes, capacidad para atender las demandas sociales, aspectos éticos y morales, grado de satisfacción de los estudiantes, contribución al desarrollo cultural y cívico, condiciones de trabajo y empleo de los docentes y movilidad académica deberían también ser consideradas ${ }^{13}$.

Así mismo, recomienda la creación de instancias nacionales independientes y definirse normas comparativas de calidad, reconocidas en el plano internacional. Con miras a tener en cuenta la diversidad y evitar la uniformidad, debería prestarse la atención debida a las particularidades de los contextos institucional, nacional y regional. Los protagonistas deben ser parte integrante del proceso de evaluación institucional $^{13}$.
El modelo ISO es normativo, prescriptivo, centrado en el proceso, en el cómo hay que hacer las cosas. Propone una serie de requisitos de obligado cumplimiento, del cumplimiento de estos requisitos se obtiene un certificado concedido por una agencia externa acreditada para ello. Es en este modelo en el que se basa el actual modelo nacional para la acreditación de carreras universitarias.

Podría dar la apariencia de una vista empresarial, donde todos los elementos están a disposición para la fabricación del producto, en nuestro caso, del egresado. Sin embargo, este modelo apunta a un ordenamiento, a un trabajo coordinado donde los diferentes elementos se interrelacionen para aunar esfuerzos. Se pretende evitar superposición de tareas y compartir los resultados. No cabe aquí un trabajo individualista; se comparte información, se comparten recursos y se requiere de un buen sistema de información y comunicación. Es imperativo contar con registros y evidencias de lo actuado, lo que puede suponer para muchos dedicar un mayor tiempo para llenar formatos e informes.

Es interesante la adaptación del concepto de calidad del ámbito empresarial al educativo. No solo la definición resulta compleja. Los estudiantes son considerados como "usuarios" porque a ellos se les brinda el servicio educativo; sin embargo, los docentes también son usuarios, así como el Estado, comunidades académicas nacionales e internacionales, instituciones privadas y la sociedad misma. Es más complejo aún, pues el estudiante además de "usuario" tiene la condición de "producto" o "servicio", porque es en él que se produce la transformación a través del proceso enseñanza aprendizaje. Cabe preguntarse, entonces, si el estudiante visto como usuario es consciente de lo que requiere como producto de la institución educativa, si es capaz de describir las especificaciones para su producto/servicio. Y cuando finaliza el proceso educativo, si está en condiciones de evaluar el producto/servicio que recibe, es decir, el desarrollo de sus propias competencias. El gráfico 1 ilustra la entrada y salida del proceso de educación superior.

Existe la tarea de adecuación de los procesos productivos o de servicio de las empresas al ámbito educativo. Si se hace una analogía a la etapa de adquisición de los insumos o materias primas de la empresa, esta equivaldría a la selección de estudiantes. En el momento actual, el proceso de selección de los estudiantes es difícil cuando existe una amplia oferta a la cual están sometidos los postulantes.

Por otro lado, no hay posibilidad de descartar los productos con errores, la Universidad debe contemplar los mecanismos de seguimiento de los estudiantes. El servicio posventa comercial, para la Universidad, equivaldría a la capacitación continua que debe ofrecer a sus egresados.

Otros elementos de "entrada" del sistema lo constituyen los profesores, además de la infraestructura, equipos, financiamiento, porque son los insumos con los que se lleva a cabo el proceso educativo. Los elementos del "proceso" educativo, es decir lo que se hace o realiza, son los procesos de enseńanza aprendizaje, investigación, extensión universitaria y proyección social como el quehacer fundamental de la vida universitaria. Los elementos de "salida”, o el resultado de los procesos, lo constituyen los egresados competentes e insertados en el mercado laboral, publicaciones científicas que impactan en el medio, actividades de educación continua a los académicos, entre otros.

Es posible enfatizar ciertos elementos evaluativos, dependiendo de los propósitos o intereses de los usuarios. Es comprensible que, por ejemplo, una agencia de gobierno o los empleadores se centren en los elementos de "salida”, en tanto que los académicos o los alumnos lo hagan en los aspectos del proceso. No cabe duda de que sería más sencillo abordar o evaluar el área de "entrada", siendo más complejo hacerlo con la de "proceso". En todo caso, los elementos son difíciles de analizar, en la práctica, de manera absolutamente aislada en una fase del sistema. 
Tabla 1. Etapas en el desarrollo y evolución del término calidad

\begin{tabular}{ll}
\hline \multicolumn{1}{c}{ ETAPAS } & \multicolumn{1}{c}{ DESCRIPCIÓN } \\
\hline 1. Trabajo artesanal & $\begin{array}{l}\text { "Hacer las cosas bien". Trabajo organizado por oficios, personas expertas en todas las actividades } \\
\text { que conformaban la producción. }\end{array}$ \\
2. Pequeño empresario & $\begin{array}{l}\text { "División del trabajo". Cada persona realiza una pequeña parte del proceso, solo el nivel más alto } \\
\text { de la organización dispone de una visión global. El aumento de la producción dio lugar a la Revolu- } \\
\text { ción industrial. } \\
\text { "Hacer las cosas bien a la primera", máxima desarrollada en Japón. Se minimiza costes y se preo- } \\
\text { cupan por la satisfacción del cliente. } \\
\text { 3. Posguerra mundial }\end{array} \quad \begin{array}{l}\text { Conjunto de actividades planeadas y sistemáticas que son necesarias para proveer una confianza } \\
\text { adecuada de que el producto o servicio va a satisfacer los requisitos de calidad. }\end{array}$ \\
5. Calidad total & $\begin{array}{l}\text { Forma de gestión de una organización basada en la participación de todos sus miembros. Pretende } \\
\text { un éxito a largo plazo mediante la satisfacción del cliente y beneficios para todos los miembros de } \\
\text { la organización y la sociedad. } \\
\text { Orientada hacia la excelencia empresarial. Apunta más allá de la calidad de los productos y de la } \\
\text { eficiencia de los procesos para fijarse en la organización en su globalidad. }\end{array}$ \\
\hline
\end{tabular}

Adaptado de Cancela A y col. La gestión de calidad ante la actual dimensión universitaria en Espańa.

Form Univ. 2010; 3(2):29-36.

\section{Gráfico 1. Modelo de calidad para la acreditación institucional universitaria Gestión del Programa}

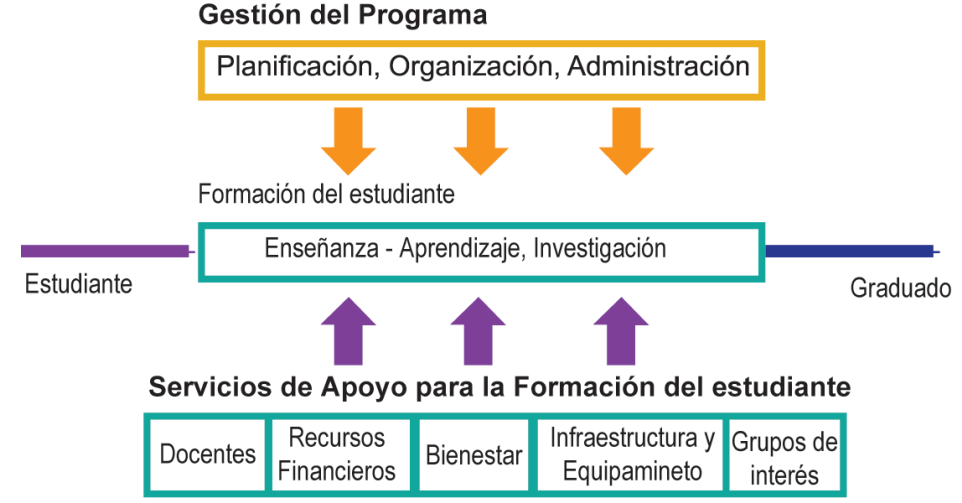

Fuente: Consejo de Evaluación, Acreditación y Certificación de la Calidad de la Educación Superior Universitaria (CONEAU). Modelo de Calidad para la Acreditación de Carreras Profesionales Universitarias, 2009.

\section{Discusión}

El tratar de definir la calidad en educación nos lleva a pensar en una educación de excelencia, con las más altas aspiraciones que puede suponer una educación privilegiada, una educación de élite. En nuestro país, las universidades se han masificado y no puede negarse que hay un mayor acceso a amplios sectores poblacionales que no proceden precisamente de esta élite. Es papel de la universidad peruana responder a este fenómeno, con equidad y pertinencia, a través de mecanismos que aseguren que los estudiantes cumplan con las exigencias que demandan sus estudios superiores.

Toda institución debe hacer un esfuerzo por definir calidad, qué características se vinculan a este concepto, cuáles son las que se acercan a este concepto y cuáles no lo son. Esta tarea involucra no solo a directivos sino también a los diferentes estamentos como profesores, estudiantes y administrativos. El punto de vista de los egresados y grupos de interés debería ser considerado. El reconocer lo que significa calidad para una institución es el punto de partida para su búsqueda permanente.

No hay claridad de qué aspectos deben ser medidos para determinar si una institución o programa es de calidad. Existen diversos modelos propuestos para este fin, pero que no necesariamente se podrían adecuar a la realidad de cada institución, sea esta pública o privada.

Es importante que los propósitos de mejora de una institución sea un proceso participativo, donde autoridades, profesores, estudiantes, trabajadores y egresados se identifiquen con la búsqueda de la mejora. Si, además, la institución opta por la acreditación, el contenido del informe de autoevaluación debe ser compartido por toda la comunidad universitaria o de la carrera de forma tal que se vean reflejados en su contenido y se identifiquen con él.

El camino hacia la calidad no es un proceso sencillo; deben producirse cambios que pueden ser dolorosos, como desprenderse de algunas prácticas o estilos para la institución y sus integrantes. Lo importante es la actitud para enfrentar este reto cuyos beneficios valen la pena asumir.

\section{Conclusión}

El término calidad en el ámbito de la educación tiene múltiples acepciones y enfoques. Las instituciones deberían definirla como punto de partida para la búsqueda de la mejora. Este proceso debe involucrar a los diferentes actores de la institución y responder a las necesidades de la sociedad en su conjunto.

\section{Referencias bibliográficas}

1. Ley No 28740, Ley del Sistema Nacional de Evaluación, Acreditación y Certificación de la Calidad Educativa. [Citado: 2014-01-10]. Disponible en: http://www.peru. gob.pe/docs/PLANES/14243/ PLAN_14243_2013_Reglamento_SINEACE_Ley_28740.pdf

2. Ley Universitaria, Ley $\mathrm{N}^{\circ} 30220$. Normas Legales El Peruano, 9 de julio 2014.

3. Cancela A, Sánchez A, Gandón R, Rey M. La gestión de calidad ante la actual dimensión universitaria en España. Form Univ. 2010; 3(2):29-36. 
4. Redon S. Autoevaluación institucional y acreditación como aseguramiento de la calidad de la educación: implicancias teóricas y prácticas. Estud Pedagog. 2009. 35(2): 269-284.

5. Pareja J. Una clave para la calidad de la institución educativa: los planes de mejora. Educ Educ. 2006; $9(2): 171-85$.

6. Guzmán J. La calidad de la enseñanza en educación superior. Perfiles Educativos. 2011; 33(número especial):129-41.

7. Vidal M, Morales I. Calidad educativa. Educ Med Super. 2010; 24(2):253-74.

8. Ministerio de Educación (MINEDU). Ley General de Educación No 28044. [Citado 2014-01-10]. Disponible en: http://www.minedu.gob.pe/normatividad/leyes.php

9. Presidencia de la República. Decreto Supremo N 018-2007-ED. Reglamento de Ley N 28740.

10. Salas R. La calidad en el desarrollo profesional: avances y desafíos. Rev Cubana Educ Med Super. 2000; 14(2):136-47.

11. Yzaguirre L. Calidad educativa e ISO 9001-2000 en México. REICE, 2005; 3(1):421-31.

12. Consejo de Evaluación, Acreditación y Certificación de la Calidad de la Educación Superior Universitaria (CONEAU). Modelo de Calidad para la Acreditación de
Carreras Profesionales Universitarias, 2009.

13. Unesco (1998). Conferencia Mundial sobre la Educación Superior: la educación superior en el siglo XXI. 1998; París: [ Citado 201307-10]. UNESCO. Disponible en: http:/www.unesco.org/education/ educprog/wche/declaration_spa. htm

14. Cuya R. Calidad relativa. [Citado 2013-07-10]. [ Citado 2013-0710]. Disponible en: http://www. calidadintegral.com/relativa.php

15. ISO. How does ISO develop standards? [Citado 2013-07-10]. Disponible en: http://www.iso.org/iso/ home/standards_development.htm

16. Rico J. Evolución del concepto de calidad. Rev Esp Trasp. 10(3):169-75. [Citado 2013-0710]. Disponible en: http://www. elmedicointeractivo.com/ap 1/ emiold/publicaciones/trasplantes3/169-175.pdf

17. Red Iberoamericana para la Acreditación de la Calidad de la Educación Superior RIACES. Glosario Internacional RIACES de la Evaluación de la Calidad y Acreditación. 2004; Madrid: RIACES.

18. Rama C, Domínguez J. El aseguramiento de la calidad de la educación virtual. 2011; Lima: Imprenta Editora Gráfica Real SAC.

19. Freire M, Teijeiro M. Revisión histórica de la garantía de calidad externa en las instituciones de educación superior. Rev Educ Sup. 2010; 34(155):123-36.

20. Henard F. Aprendamos la lección.Un repaso a la calidad de la enseñanza en la educación superior. Perfiles Educativos. 2010; 32(129):164-73.

21. Pires S, Lemaitre J. Sistemas de Acreditación y Evaluación de la Educación Superior en América Latina y El Caribe. Tendencias de la Educación Superior en América Latina y el Caribe. 2008; Caracas: Instituto Internacional de la Unesco para la Educación Superior en América Latina y el Caribe.

22. Consejo Nacional de Acreditación de Colombia (CNA). Lineamientos para la acreditación de programas de pregrado, 2013.

23. Comisión Nacional de Evaluación y Acreditación Universtiaria (CONEAU Argentina). Acreditación de carreras de grado de interés público. Guía de autoevaluación, 2007.

24. Comisión Nacional de Acreditación de Chile. Guía para la acreditación. Normas y procedimientos, 2007.

25. European Association for Quality Assurance in Higher Education (ENQA). Criterios y Directrices para la Garantía de Calidad en el Espacio Europeo de Educación Superior, 2005. [Citado 2014-11-22] Disponible en http://www.enqa. net/bologna.lasso 